Article

\title{
Weighted Method for Uncertain Nonlinear Variational Inequality Problems
}

\author{
Cunlin Li $1,2,3,+\left(\mathbb{D}\right.$, Mihai Postolache ${ }^{4,5,6, *,+}$ and Zhifu Jia ${ }^{2,3,+}$ (i) \\ 1 Ningxia Key Laboratory of Intelligent Information and Big Data Processing, Governance and Social \\ Management Research Center of Northwest Ethnic Regions, North Minzu University, \\ Yinchuan 750021, China; bitlcl@163.com \\ 2 State Key Laboratory of Mechanics Control of Mechanical Structures, Institute of Nano Science and \\ Department of Mathematics, Nanjing University of Aeronautics and Astronautics, Nanjing 210016, China; \\ jzflzbx@163.com \\ 3 School of Mathematics and Information Science, North Minzu University, Yinchuan 750021, China \\ 4 Center for General Education, China Medical University, Taichung 40402, Taiwan \\ 5 Institute of Mathematical Statistics and Applied Mathematics, Romanian Academy, \\ 050711 Bucharest, Romania \\ 6 Department of Mathematics and Informatics, University "Politehnica" of Bucharest, 060042 Bucharest, Romania \\ * Correspondence: mihai@mathem.pub.ro \\ + These authors contributed equally to this work.
}

Received: 19 August 2019; Accepted: 10 October 2019; Published: 15 October 2019

check for updates

\begin{abstract}
A convex combined expectations regularized gap function with uncertain variable is presented to deal with uncertain nonlinear variational inequality problems (UNVIP). The UNVIP is transformed into a minimization problem through an uncertain weighted expected residual function. Moreover, the convergence of the global optimal solutions of the uncertain weighted expected residual minimization model is given through the integration by parts method under the compact space of the uncertain event. The limiting behaviors of the transformed model are analyzed. Furthermore, a compact approximation method is proposed in the unbounded uncertain event space. Through analysis of the convergence of UWERM model and reasonable hypothesis, the compact approximation method is verified under the circumstance of Holder continuity.
\end{abstract}

Keywords: uncertain variational inequality; UWERM problem; convergence analysis

MSC: 49J53; 49J40; 65K10; 90C99

\section{Introduction}

Let $S \in \mathbb{R}^{n}$ be a nonempty closed convex set and $f: S \rightarrow \mathbb{R}^{n}$ be a mapping. If there is a vector $x^{*} \in S$ such that

$$
\left(x-x^{*}\right)^{T} f\left(x^{*}\right) \geq 0, \quad x \in S
$$

holds, it is called the variational inequality problem (denoted by $\operatorname{VIP}(f, S)$ ). In the past several years, the VIP is always a very hot problem in the field of the operation research. The developments of VIP involve theoretic research, effective algorithm for finding solution and applications. Yao [1] established a generalized quasi-variational inequality model. The study of the existence of the variational inequality problem is extended in finite dimensional spaces and the class of problems modeled by the variational inequality theory is enlarged. Some significant existence results are established based on mild assumptions without convexity. In [2], the existence of the variational and the generalized variational inequality problems are presented under the discontinuous mappings. 
The uniqueness of the solution of the generalized variational inequality problem is given and the existence of the generalized complementarity problem is investigated. In [3], they study the existence of the set-valued variational inequalities (VVI) based on vector mappings. The necessary and sufficient conditions of the existence are established based on the gap functions of VVI. The above shows that the VIP can be transformed into a semi-infinite programming problem by a proper gap function. Postolache et al. [4-7] made a contribution to the solution and application of variational inequalities. In [4], they consider three classes of variational problem including multitime multiobjective variational problem (VVP), multitime vector fractional variational problem (VFP), and multitime scalar variational problem (SVP). The necessary optimality conditions are established for SVP. The efficient solution and the normal efficiency solution of the two vector variational problems VVP and VFP are presented, and necessary efficiency of conditions of the efficient solution and the normal efficiency solution are established. In [5], the authors construct algorithms for a class of monotone variational inequalities. In [6], the writers prove the existence and approximation of solutions for generalized extended nonlinear variational inequalities. In [7], the authors propose the variant extragradient-type method for monotone variational inequalities. Yao et al. have done a lot of research into the variational inequality problem in the last few years (see [8-10]); also, Facchinei and Pang [11] have done similar research. However, for Variational Inequalities with uncertain variables, they involve very little. Uncertainty theory [12] models a kind of degree of belief that the uncertain event will happen. Many applications also contain some uncertain variable, such as a new stock, emergencies, devastating military experiments, etc. Subsequently, the uncertain variational inequality problem is proposed by Chen and Zhu [13]. In their paper, a new class of uncertain variational inequality problems are proposed to find $x^{*} \in S$ such that

$$
\mathscr{M}\left\{\gamma \in \Gamma \mid\left(x-x^{*}\right)^{T} F\left(x^{*}, \xi(\gamma)\right) \geq 0, \forall x \in S\right\}=1,
$$

where $\xi(\gamma)$ is the uncertain variable, $\Gamma$ is a nonempty set, $F: \mathbb{R}^{n} \times \Gamma \rightarrow \mathbb{R}^{n}$ is a mapping. They solve the expected value model based on uncertainty theory.

$\mathrm{Li}$ and Jia [14] introduced the uncertain variable in the VIP model, they established an expected residual model about the uncertain variable through a regularized gap function. It is given as

$$
\begin{aligned}
& \min \theta(x) \\
& \theta(x):=E[g(x, \xi)]=\int_{T} g(x, t) d \Phi(t) \\
& \text { s.t. } x \in S,
\end{aligned}
$$

here, $\xi \in B, E$ stands for the expectation with respect to the uncertain variable $\xi, \Phi(t)$ stands for the uncertain distribution function with respect to the uncertain variable $\xi$. T stands for domain of $\Phi(t)$. Recall that, for $\forall x \in \mathbb{R}^{n}$ and $\forall \xi \in B$,

$$
g(x, \xi)=(x-H(x, \xi))^{T} F(x, \xi)-\frac{\alpha}{2}\|x-H(x, \xi)\|_{G^{\prime}}^{2}
$$

where

$$
H(x, \xi):=\operatorname{Proj}_{S . G}\left(x-\alpha^{-1} G^{-1} F(x, \xi)\right)
$$

$F: \mathbb{R}^{n} \times \mathbb{R} \rightarrow \mathbb{R}^{n}$ is a mapping. $\alpha$ is a positive parameter, $G$ is an $n \times n$ symmetric positive-definite matrix, and $\|\cdot\|_{G}$ means the G-norm defined by $\|x\|_{G}=\sqrt{x^{T} G x}$ for $x \in \mathbb{R}^{n}$.

In [14], Li and Jia considered a linear uncertain variational inequality problem. The properties and convergence analysis of the ERM problem were discussed. Integration by parts method is proposed to solve (2). The purpose of this paper is to introduce UWERM model for dealing with nonlinear uncertain variational inequality problem. 
The paper is organized as following. We recall some preliminary results about uncertainty theory and other preliminaries in Section 2. Then, the convergence of global optimal solutions and convergence of stationary points of UWERM model are discussed in Section 3. Furthermore, the compact approximations of UWERM model are covered in Section 4. Finally, the conclusions are given in Section 5.

\section{Preliminaries}

\subsection{Uncertainty Theory}

In this section, some fundamental concepts, properties concerning uncertain variables, uncertain distribution and expectation are recalled. Let $\Gamma$ be a nonempty set, and $\mathscr{L}$ a $\sigma$-algebra over $\Gamma$. Each element $\Lambda$ in $\mathscr{L}$ is called an event and assigned a number $\mathscr{M}\{\Lambda\}$ to indicate the belief degree with which the $\Lambda$ will happen. In order to deal with belief degrees correctly, Liu put forward the following three axioms: Axiom 1 (Normality Axiom). $\mathscr{M}\{\Gamma\}=1$ for the universal set $\Gamma$;

Axiom 2 (Duality Axiom). $\mathscr{M}\{\Lambda\}+\mathscr{M}\left\{\Lambda^{c}\right\}=1$ for any event $\Lambda$;

Axiom 3 (Subadditivity Axiom). For each countable sequence of events $\Lambda_{1}, \Lambda_{2}, \ldots$, we have

$$
\mathscr{M}\left\{\bigcup_{i=1}^{\infty} \Lambda_{i}\right\} \leq \sum_{i=1}^{\infty} \mathscr{M}\left\{\Lambda_{i}\right\} .
$$

Definition 1 (Liu [12]). The set function $\mathscr{M}$ is called an uncertain measure if it satisfies the normality, duality, and subadditivity axioms.

The triplet $(\Gamma, \mathscr{L}, \mathscr{M})$ is called an uncertainty space. Furthermore, the product uncertain measure on the product $\sigma$-algebra $\mathscr{L}$ was defined by Liu as follows:

Axiom 4 (Product Axiom). Let $\left(\Gamma_{k}, \mathscr{L}_{k}, \mathscr{M}_{k}\right)$ be uncertainty spaces for $k=1,2, \ldots$. The product uncertain measure $\mathscr{M}$ is an uncertain measure satisfying

$$
\mathscr{M}\left\{\prod_{k=1}^{\infty} \Lambda_{k}\right\}=\bigwedge_{k=1}^{\infty} \mathscr{M}_{k}\left\{\Lambda_{k}\right\}
$$

where $\Lambda_{k}$ are arbitrary events chosen from $\mathscr{L}_{k}$ for $k=1,2, \ldots$, respectively.

Definition 2 (Liu [12]). An uncertain variable is a measurable function $\xi$ from an uncertainty space $(\Gamma, \mathscr{L}, \mathscr{M})$ to the set of real numbers, i.e., for any Borel set B of real numbers, the set

$$
\{\xi \in B\}=\{\gamma \in \Gamma \mid \xi(\gamma) \in B\}
$$

is an event.

Theorem 1 (Liu [12]). Let $\xi_{1}, \xi_{2}, \ldots, \xi_{n}$ be uncertain variables, and $f$ a real-valued measurable function. Then $\xi=f\left(\xi_{1}, \xi_{2}, \ldots, \xi_{n}\right)$ is an uncertain variable defined by

$$
\xi(\gamma)=f\left(\xi_{1}(\gamma), \xi_{2}(\gamma), \ldots, \xi_{n}(\gamma)\right), \quad \forall \gamma \in \Gamma
$$

Definition 3 (Liu [12]). Suppose $\xi$ is an uncertain variable. Then the uncertainty distribution of $\xi$ is defined by

$$
\Phi(t)=\mathscr{M}\{\xi \leq t\}
$$

for any real number $t$.

For ranking uncertain variables, the concept of expected value was proposed by Liu [12] as follows: 
Definition 4 (Liu [12]). Let $\xi$ be an uncertain variable. Then the expected value of $\xi$ is defined by

$$
E[\xi]=\int_{0}^{+\infty} \mathscr{M}\{\xi \geq t\} d t-\int_{-\infty}^{0} \mathscr{M}\{\xi \leq t\} d t
$$

provided that at least one of the two integrals is finite.

Theorem 2 (Liu [12]). Let $\xi$ be an uncertain variable with uncertainty distribution $\Phi$. If the expected value exists, then

$$
E[\xi]=\int_{-\infty}^{+\infty} t d \Phi(t)
$$

Corollary 1 (Liu [12]). Let $\xi$ be an uncertain variable with uncertainty distribution $\Phi$ and let $f(t)$ be a strictly monotone function, then we have

$$
E[f(\xi)]=\int_{-\infty}^{+\infty} f(t) d \Phi(t)
$$

\subsection{Other Preliminaries}

From Theorem 4.2 of [14] and the continuity of $\left(F, \nabla_{x} F\right), \theta$ is continuously differentiable over $S$ and

$$
\nabla \theta(x)=E\left[\nabla_{x} g(x, \xi)\right], \forall x \in S .
$$

From non-additivity of the uncertain variable, we can tell there is no density function for uncertain variable. So $\Phi(x)$ usually is not differentiable in uncertainty theory. By the results given in [15], $g(\cdot, \xi)$ is a continuously differentiable function over $S$ for any $\xi \in B$, and

$$
\nabla_{x} g(x, \xi)=F(x, \xi)-\left(\nabla_{x} F(x, \xi)-\alpha G\right)(H(x, \xi)-x) .
$$

Theorem 3. Through [16], we can get the following conclusion

$$
\lim _{k \rightarrow \infty} \frac{1}{N_{k}} \sum_{t_{i} \in T_{k}} \Phi\left(t_{i}\right) g^{\prime}\left(x, t_{i}\right)=\int_{T} \Phi(t) g^{\prime}(x, t) d t
$$

For any $x \in \mathbb{R}^{n}$, we also have

$$
\sqrt{\lambda_{\min }}\|x\| \leq\|x\|_{G} \leq \sqrt{\lambda_{\max }}\|x\|
$$

where $G$ is a positive definite matrix, $\lambda_{\min }$ and $\lambda_{\max }$ are its smallest and largest eigenvalues, respectively. $\|A\|$ and $\|A\|_{\mathscr{F}}$ denote the spectral norm and the Frobenius norm of matrix $\mathrm{A}$, respectively. The relationship between $\|A\|$ and $\|A\|_{\mathscr{F}},\|x\|$ and $\|x\|_{G}$ are as follows,

$$
\begin{gathered}
\|A\| \leq\|A\|_{\mathscr{F} .} \\
\|A\|_{\mathscr{F}} \leq \sum_{j=1}^{n}\left\|A_{j}\right\| .
\end{gathered}
$$

where $A_{j}$ is the jth column vector of $A$.

These definitions and properties will be used in the latter theorem.

\section{Convergence Analysis}

\subsection{UWERM Establishment and Hypothesis}

In this paper, the next formulation (10) is called a uncertain weighted expected residual minimization model with $\lambda \in[0,1]$. 


$$
\begin{aligned}
\min \theta(x): & =\lambda E[g(x, \xi)]+(1-\lambda) E\left[g^{2}(x, \xi)\right] \\
& =\int_{T}\left(\lambda g(x, t)+(1-\lambda) g^{2}(x, t)\right) d \Phi(t) \\
& \text { s.t. } x \in S
\end{aligned}
$$

where $g$ is defined as (3), $E$ stands for the expectation with respect to the uncertain variable $\xi, \Phi(t)$ stands for the uncertain distribution function with respect to the uncertain variable $\xi$.

In this section, we assume that the uncertain space $T$ is compact. Under this assumption, we will investigate the convergence results for (10).

Definition 5. Let $\theta_{k}(x)$ minimum be as follows:

$$
\begin{aligned}
& \min \theta_{k}(x)=\left.\lambda g(x, t) \Phi(t)\right|_{t \in T}-\frac{\lambda}{N_{k}} \sum_{t_{i} \in T_{k}} \Phi\left(t_{i}\right) g^{\prime}\left(x, t_{i}\right) \\
& +\left.(1-\lambda) g^{2}(x, t) \Phi(t)\right|_{t \in T}-\frac{2(1-\lambda)}{N_{k}} \sum_{t_{i} \in T_{k}} \Phi\left(t_{i}\right) g\left(x, t_{i}\right) g^{\prime}\left(x, t_{i}\right)
\end{aligned}
$$

where the sets $T_{k}=\left\{t_{i} \mid i=1,2, \ldots, N_{k}\right\}$ is generated by [16], and satisfy $N_{k} \rightarrow \infty$ as $k \rightarrow \infty$, we call $T$ uncertain event space, theoretically $T$ is domain of $\Phi(t),\left.g(x, t) \Phi(t)\right|_{t \in T}$ is a function merely related to $x$.

We will study the following approximations problem to the UWERM problem (10) as follows:

$$
\begin{aligned}
& \min \theta_{k}(x)=\left.\lambda g(x, t) \Phi(t)\right|_{t \in T}-\frac{\lambda}{N_{k}} \sum_{t_{i} \in T_{k}} \Phi\left(t_{i}\right) g^{\prime}\left(x, t_{i}\right) \\
& +\left.(1-\lambda) g^{2}(x, t) \Phi(t)\right|_{t \in T} \\
& -\frac{2(1-\lambda)}{N_{k}} \sum_{t_{i} \in T_{k}} \Phi\left(t_{i}\right) g\left(x, t_{i}\right) g^{\prime}\left(x, t_{i}\right) \\
& \text { s.t. } x \in S
\end{aligned}
$$

We study the limiting problems (12) in the next section. Let the function $F$ be affine. Some assumptions such as the positive definiteness and the square integrability are given, see [14] for details. To prove the latter theorem, we suppose that the uncertain distribution function $\Phi(t)$ is continuous on $t \in T$, and that a third order derivative of the function $F$ exists (denoted by $F_{x t x}^{\prime \prime \prime}$ ). Owing to $H(x, t):=\operatorname{Proj}_{S . G}\left(x-\alpha^{-1} G^{-1} F(x, t)\right)$ and $H^{\prime}(x, t):=\operatorname{Proj}_{S . G}\left(x-\alpha^{-1} G^{-1} F^{\prime}(x, t)\right)$, it is easy to get $H$, $H_{x}^{\prime}$, and $H_{t}^{\prime}$ are continuous. First, the convergence of global optimal solutions is considered.

\subsection{Convergence of Global Optimal Solutions}

For convenience, we denote by $S^{*}$ and $S_{k}^{*}$ the sets of optimal solutions of problems (10) and (12), respectively. Now, we first give the following lemma which is the robust convergence version of ERM approximate problem.

Lemma 1. For any fixed $x \in S$, we have

$$
\theta(x)=\lim _{k \rightarrow \infty} \theta_{k}(x)
$$

Proof. It is known from the definition of $\theta(x)$ function of (10) and integration by parts method that 


$$
\begin{aligned}
\theta(x): & =\lambda E[g(x, \xi)]+(1-\lambda) E\left[g^{2}(x, \xi)\right] \\
& =\int_{T}\left(\lambda g(x, t)+(1-\lambda) g^{2}(x, t)\right) d \Phi(t) \\
& =\left.\lambda g(x, t) \Phi(t)\right|_{t \in T}-\lambda \int_{T} \Phi(t) g^{\prime}(x, t) d t \\
& +\left.(1-\lambda) g^{2}(x, t) \Phi(t)\right|_{t \in T} \\
& -(1-\lambda) \int_{T} \Phi(t) 2 g(x, t) g^{\prime}(x, t) d t
\end{aligned}
$$

while by the definition of $\theta_{k}(x)$ function of (11) that

$$
\begin{aligned}
& \theta_{k}(x)=\left.\lambda g(x, t) \Phi(t)\right|_{t \in T}-\frac{\lambda}{N_{k}} \sum_{t_{i} \in T_{k}} \Phi\left(t_{i}\right) g^{\prime}\left(x, t_{i}\right) \\
& +\left.(1-\lambda) g^{2}(x, t) \Phi(t)\right|_{t \in T}-\frac{2(1-\lambda)}{N_{k}} \sum_{t_{i} \in T_{k}} \Phi\left(t_{i}\right) g\left(x, t_{i}\right) g^{\prime}\left(x, t_{i}\right)
\end{aligned}
$$

It can be straightforwardly obtained from (6) that

$$
\lim _{k \rightarrow \infty} \frac{1}{N_{k}} \sum_{t_{i} \in T_{k}} \Phi\left(t_{i}\right) g^{\prime}\left(x, t_{i}\right)=\int_{T} \Phi(t) g^{\prime}(x, t) d t
$$

and

$$
\lim _{k \rightarrow \infty} \frac{1}{N_{k}} \sum_{t_{i} \in T_{k}} \Phi\left(t_{i}\right) g\left(x, t_{i}\right) g^{\prime}\left(x, t_{i}\right)=\int_{T} \Phi(t) g(x, t) g^{\prime}(x, t) d t .
$$

Thus it holds that $\theta(x)=\lim _{k \rightarrow \infty} \theta_{k}(x)$.

Theorem 4. Assume that $x_{k} \in S_{k}^{*}$ for each sufficiently large $k$. and $x^{*}$ is an accumulation point of $\left\{x_{k}\right\}$. Then, we have $x^{*} \in S^{*}$

Proof. Let $x^{*}$ be an accumulation point of $\left\{x_{k}\right\}$. Without loss of generality, we assume that $\left\{x_{k}\right\}$ converges to $x^{*}$. It is obvious that $x^{*} \in S$. We first show that

$$
\lim _{k \rightarrow \infty}\left(\theta_{k}\left(x_{k}\right)-\theta_{k}\left(x^{*}\right)\right)=0 .
$$

Let $B \subset S$ be a compact convex set containing the sequence $x_{k}$. By the continuity of $g_{t x}^{\prime \prime}, g^{\prime}$ and $g$ on the compact set $B \times T$, there exists a constant $C_{1}, C_{2}, C_{3}>0$ such that

$$
\begin{gathered}
\left\|g_{t x}^{\prime \prime}(x, t)\right\| \leq C_{1}, \forall(x, \xi) \in B \times T . \\
\left\|g^{\prime}(x, t)\right\| \leq C_{2}, \forall(x, \xi) \in B \times T . \\
\|g(x, t)\| \leq C_{3}, \forall(x, \xi) \in B \times T .
\end{gathered}
$$

Moreover, we have from the mean-value theorem that, for each $x_{k}$ and each $\xi_{i}$, there exists $y^{k i}=$ $\alpha_{k i} x_{k}+\left(1-\alpha_{k i}\right) x^{*} \in B$ with $\alpha_{k i} \in[0,1]$ such that

$$
\left|g_{t}^{\prime}\left(x_{k}, t_{i}\right)-g_{t}^{\prime}\left(x^{*}, t_{i}\right)\right|=\left|g_{t x}^{\prime \prime}\left(y^{k i}, t_{i}\right)^{T}\left(x_{k}-x^{*}\right)\right|
$$

also there exists $z_{k i}=\beta_{k i} x_{k}+\left(1-\beta_{k i}\right) x^{*} \in B$ with $\beta_{k i} \in[0,1]$ such that

$$
\begin{gathered}
\left|\left(g\left(x_{k}, t\right) g_{t}^{\prime}\left(x_{k}, t_{i}\right)-g\left(x^{*}, t\right) g_{t}^{\prime}\left(x^{*}, t_{i}\right)\right)\right| \\
=\left|\left(g_{x}^{\prime} g_{t}^{\prime}+g g_{t x}^{\prime \prime}\right)\left(z_{k i}, t_{i}\right)^{T}\left(x_{k}-x^{*}\right)\right|
\end{gathered}
$$


So, we have

$$
\begin{aligned}
& \left|\theta_{k}\left(x_{k}\right)-\theta_{k}\left(x^{*}\right)\right| \\
& =\left|\lambda g\left(x_{k}, t\right) \Phi(t)\right|_{t \in T}-\frac{\lambda}{N_{k}} \sum_{t_{i} \in T_{k}} \Phi\left(t_{i}\right) g_{t}^{\prime}\left(x_{k}, t_{i}\right) \\
& +\left.(1-\lambda) g^{2}\left(x_{k}, t\right) \Phi(t)\right|_{t \in T} \\
& -\frac{1-\lambda}{N_{k}} \sum_{t_{i} \in T_{k}} 2 \Phi\left(t_{i}\right) g\left(x_{k}, t\right) g_{t}^{\prime}\left(x_{k}, t_{i}\right) \\
& -\left.\lambda g\left(x^{*}, t\right) \Phi(t)\right|_{t \in T}+\frac{\lambda}{N_{k}} \sum_{t_{i} \in T_{k}} \Phi\left(t_{i}\right) g_{t}^{\prime}\left(x^{*}, t_{i}\right) \\
& -\left.(1-\lambda) g^{2}\left(x^{*}, t\right) \Phi(t)\right|_{t \in T} \\
& +\frac{1-\lambda}{N_{k}} \sum_{t_{i} \in T_{k}} \Phi\left(t_{i}\right) 2 g\left(x^{*}, t\right) g_{t}^{\prime}\left(x^{*}, t_{i}\right) \\
& \leq\left|\lambda g\left(x_{k}, t\right) \Phi(t)\right|_{t \in T}-\left.\lambda g\left(x^{*}, t\right) \Phi(t)\right|_{t \in T} \mid \\
& +\left|(1-\lambda) g^{2}\left(x_{k}, t\right) \Phi(t)\right|_{t \in T}-\left.(1-\lambda) g^{2}\left(x^{*}, t\right) \Phi(t)\right|_{t \in T} \mid \\
& +\left|\frac{\lambda}{N_{k}} \sum_{t_{i} \in T_{k}} \Phi\left(t_{i}\right)\left(g_{t}^{\prime}\left(x_{k}, t_{i}\right)-g_{t}^{\prime}\left(x^{*}, t_{i}\right)\right)\right| \\
& +\left|\frac{1-\lambda}{N_{k}} \sum_{t_{i} \in T_{k}} 2 \Phi\left(t_{i}\right)\left(g\left(x_{k}, t\right) g_{t}^{\prime}\left(x_{k}, t_{i}\right)-g\left(x^{*}, t\right) g_{t}^{\prime}\left(x^{*}, t_{i}\right)\right)\right| \\
& \leq\left|\lambda g\left(x_{k}, t\right) \Phi(t)\right|_{t \in T}-\left.\lambda g\left(x^{*}, t\right) \Phi(t)\right|_{t \in T} \mid \\
& +\left|(1-\lambda) g^{2}\left(x_{k}, t\right) \Phi(t)\right|_{t \in T}-\left.(1-\lambda) g^{2}\left(x^{*}, t\right) \Phi(t)\right|_{t \in T} \mid \\
& +\frac{\lambda}{N_{k}} \sum_{t_{i} \in T_{k}} \Phi\left(t_{i}\right)\left|\left(g_{t}^{\prime}\left(x_{k}, t_{i}\right)-g_{t}^{\prime}\left(x^{*}, t_{i}\right)\right)\right| \\
& +\frac{1-\lambda}{N_{k}} \sum_{t_{i} \in T_{k}} \Phi\left(t_{i}\right)\left|\left(g\left(x_{k}, t\right) g_{t}^{\prime}\left(x_{k}, t_{i}\right)-g\left(x^{*}, t\right) g_{t}^{\prime}\left(x^{*}, t_{i}\right)\right)\right| \\
& =\left|\lambda g\left(x_{k}, t\right) \Phi(t)\right|_{t \in T}-\left.\lambda g\left(x^{*}, t\right) \Phi(t)\right|_{t \in T} \mid \\
& +\left|(1-\lambda) g^{2}\left(x_{k}, t\right) \Phi(t)\right|_{t \in T}-\left.(1-\lambda) g^{2}\left(x^{*}, t\right) \Phi(t)\right|_{t \in T} \mid \\
& +\frac{\lambda}{N_{k}} \sum_{t_{i} \in T_{k}} \Phi(t)\left|g_{t x}^{\prime \prime}\left(y_{k i}, t_{i}\right)^{T}\left(x_{k}-x^{*}\right)\right| \\
& +\frac{1-\lambda}{N_{k}} \sum_{t_{i} \in T_{k}} \Phi(t)\left|\left(g_{x}^{\prime} g_{t}^{\prime}+g g_{t x}^{\prime \prime}\right)\left(z_{k i}, t_{i}\right)^{T}\left(x_{k}-x^{*}\right)\right| \\
& \leq\left|\lambda g\left(x_{k}, t\right) \Phi(t)\right|_{t \in T}-\left.\lambda g\left(x^{*}, t\right) \Phi(t)\right|_{t \in T} \mid \\
& +\left|(1-\lambda) g^{2}\left(x_{k}, t\right) \Phi(t)\right|_{t \in T}-\left.(1-\lambda) g^{2}\left(x^{*}, t\right) \Phi(t)\right|_{t \in T} \mid \\
& +\frac{\lambda}{N_{k}} \sum_{t_{i} \in T_{k}} \Phi\left(t_{i}\right)\left\|g_{t x}^{\prime \prime}\left(y_{k i}, t_{i}\right)\right\| \cdot\left\|\left(x_{k}-x^{*}\right)\right\| \\
& +\frac{1-\lambda}{N_{k}} \sum_{t_{i} \in T_{k}} \Phi\left(t_{i}\right)\left\|\left(g_{x}^{\prime} g_{t}^{\prime}+g g_{t x}^{\prime \prime}\right)\left(z_{k i}, t_{i}\right)\right\| \cdot\left\|\left(x_{k}-x^{*}\right)\right\| \\
& \leq\left|\lambda g\left(x_{k}, t\right) \Phi(t)\right|_{t \in T}-\left.\lambda g\left(x^{*}, t\right) \Phi(t)\right|_{t \in T} \mid \\
& +\left|(1-\lambda) g^{2}\left(x_{k}, t\right) \Phi(t)\right|_{t \in T}-\left.(1-\lambda) g^{2}\left(x^{*}, t\right) \Phi(t)\right|_{t \in T} \mid \\
& +C_{1}\left\|\left(x_{k}-x^{*}\right)\right\| \frac{\lambda}{N_{k}} \sum_{t_{i} \in T_{k}} \Phi\left(t_{i}\right) \\
& +\left(C_{2}^{2}+C_{1} C_{3}\right)\left\|\left(x_{k}-x^{*}\right)\right\| \frac{1-\lambda}{N_{k}} \sum_{t_{i} \in T_{k}} \Phi\left(t_{i}\right)
\end{aligned}
$$


Owing to $\sum_{t_{i} \in T_{k}} \Phi\left(t_{i}\right) \leq N_{k}$, so $\frac{1}{N_{k}} \sum_{t_{i} \in T_{k}} \Phi\left(t_{i}\right) \leq 1$. Because of the fact that the sequence $\left\{x_{k}\right\}$ converges to $\left\{x^{*}\right\}$. Therefore,

$$
\left|\theta_{k}\left(x_{k}\right)-\theta_{k}\left(x^{*}\right)\right| \stackrel{k \rightarrow \infty}{\longrightarrow} 0
$$

On the other hand, noting that

$$
\left|\theta_{k}\left(x_{k}\right)-\theta\left(x^{*}\right)\right| \leq\left|\theta_{k}\left(x_{k}\right)-\theta_{k}\left(x^{*}\right)\right|+\left|\theta_{k}\left(x^{*}\right)-\theta\left(x^{*}\right)\right|,
$$

so, we have from Lemma 1 and (16) that

$$
\lim _{k \rightarrow \infty} \theta_{k}\left(x_{k}\right)=\theta\left(x^{*}\right) .
$$

Since, for each sufficiently large $\mathrm{k}, x_{k} \in S_{k}^{*}$, there exist $\epsilon>0$ such that

$$
\theta_{k}\left(x_{k}\right) \leq \theta_{k}(x)+\epsilon
$$

holds for any $x \in S$. Letting $k \rightarrow+\infty$ in (19) and taking (18) and lemma 1 into account, we get

$$
\theta\left(x^{*}\right) \leq \theta(x)+\epsilon, \forall x \in S
$$

which means $x^{*} \in S^{*}$.

3.3. Convergence of Stationary Points

Theorem 5. If $\lim _{k \rightarrow \infty} x_{k}=x^{*}$, then

$$
\lim _{k \rightarrow \infty} \nabla \theta_{k}\left(x_{k}\right)=\nabla \theta\left(x^{*}\right)
$$

Proof. Let $B \subseteq S$ be a compact convex set containing the sequence $x_{k}$. By the continuity of $F_{t}^{\prime}, F_{t x}^{\prime \prime}, g_{t}^{\prime}, g_{t x}^{\prime \prime} H, H_{t}^{\prime}$, and $\nabla_{x}^{2} F_{j}$ on the compact set $B \times T$, there exists a constant $C \geq \sup \left\{\left\|x_{k}\right\|, k=\right.$ $1,2, \ldots\}$ such that, for any $(x, t) \in B \times T$,

$$
\begin{gathered}
\left\|F_{t}^{\prime}\right\| \leq C \\
\left\|F_{t x}^{\prime \prime}\right\| \leq C \\
\left\|g_{t}^{\prime}\right\| \leq C \\
\left\|g_{t x}^{\prime \prime}\right\| \leq C \\
\left\|F_{x t x}^{\prime \prime \prime}\right\| \leq C \\
\|H\| \leq C \\
\left\|H_{t}^{\prime}\right\| \leq C
\end{gathered}
$$

where $F_{t x}^{\prime \prime}$ denotes the derivative of $F(x, t)$ with respect to $\mathrm{x}, \mathrm{t}$. We first show that

$$
\lim _{k \rightarrow 0} \frac{\lambda}{N_{k}} \sum_{t_{i} \in T_{k}} \Phi\left(t_{i}\right)\left\|F_{t}^{\prime}\left(x_{k}, t_{i}\right)-F_{t}^{\prime}\left(x^{*}, t_{i}\right)\right\|=0 .
$$


In fact, from (8) and (9), we have

$$
\begin{aligned}
& \frac{1}{N_{k}} \sum_{t_{i} \in T_{k}} \Phi\left(t_{i}\right)\left\|F_{t}^{\prime}\left(x_{k}, t_{i}\right)-F_{t}^{\prime}\left(x^{*}, t_{i}\right)\right\| \\
\leq & \frac{1}{N_{k}} \sum_{t_{i} \in T_{k}} \Phi\left(t_{i}\right)\left\|F_{t}^{\prime}\left(x_{k}, t_{i}\right)-F_{t}^{\prime}\left(x^{*}, t_{i}\right)\right\| \mathscr{F} \\
\leq & \sum_{j=1}^{n} \frac{1}{N_{k}} \sum_{t_{i} \in T_{k}} \Phi\left(t_{i}\right)\left\|F_{t}^{\prime}\left(x_{k}, t_{i}\right)_{j}-F_{t}^{\prime}\left(x^{*}, t_{i}\right)_{j}\right\| .
\end{aligned}
$$

Moreover, for each $x_{k}, \xi_{i}$ and any fixed $j$, from the mean-value theorem, there exists $y_{k i j}=\tau_{k i j} x_{k}+$ $\left(1-\tau_{k i j}\right) \bar{x} \in B$ with $\tau_{k i j} \in[0,1]$ such that

$$
\begin{aligned}
& \frac{1}{N_{k}} \sum_{t_{i} \in T_{k}} \Phi\left(t_{i}\right)\left\|F_{t}^{\prime}\left(x_{k}, t_{i}\right)_{j}-F_{t}^{\prime}\left(x^{*}, t_{i}\right)_{j}\right\| \\
\leq & \frac{1}{N_{k}} \sum_{t_{i} \in T_{k}} \Phi\left(t_{i}\right)\left\|F_{t x}^{\prime \prime}\left(y_{k i j}, t_{i}\right)\right\|\left\|x_{k}-x^{*}\right\| \\
\leq & C\left\|x_{k}-x^{*}\right\| \frac{1}{N_{k}} \sum_{t_{i} \in T_{k}} \Phi\left(t_{i}\right) \\
& \stackrel{k \rightarrow \infty}{\longrightarrow} 0,
\end{aligned}
$$

where the second inequality follows from (21), while (27) holds immediately from (28) and (29). In a similar way, it holds that

$$
\lim _{k \rightarrow 0} \frac{\lambda}{N_{k}} \sum_{t_{i} \in T_{k}} \Phi\left(t_{i}\right)\left\|F\left(x_{k}, t_{i}\right)-F\left(x^{*}, t_{i}\right)\right\|=0
$$

and

$$
\lim _{k \rightarrow 0} \frac{\lambda}{N_{k}} \sum_{t_{i} \in T_{k}} \Phi\left(t_{i}\right)\left\|F_{x}^{\prime}\left(x_{k}, t_{i}\right)-F_{x}^{\prime}\left(x^{*}, t_{i}\right)\right\|=0
$$

and

$$
\lim _{k \rightarrow 0} \frac{\lambda}{N_{k}} \sum_{t_{i} \in T_{k}} \Phi\left(t_{i}\right)\left\|F_{x t}^{\prime \prime}\left(x_{k}, t_{i}\right)-F_{x t}^{\prime \prime}\left(x^{*}, t_{i}\right)\right\|=0
$$

It then follows from (7), (30), and the non-expansive property of $\operatorname{Proj}_{S, G}$ that

$$
\begin{aligned}
& \frac{1}{N_{k}} \sum_{t_{i} \in T_{k}} \Phi\left(t_{i}\right)\left\|H\left(x^{*}, t_{i}\right)-H\left(x_{k}, t_{i}\right)\right\| \\
\leq & \lambda_{\min }^{-\frac{1}{2}} \frac{1}{N_{k}} \sum_{t_{i} \in T_{k}} \Phi\left(t_{i}\right)\left\|H\left(x^{*}, t_{i}\right)-H\left(x_{k}, t_{i}\right)\right\|_{G} \\
\leq & \lambda_{\min }^{-\frac{1}{2}} \frac{1}{N_{k}} \sum_{t_{i} \in T_{k}} \Phi\left(t_{i}\right) \|\left(x^{*}-\alpha^{-1} G^{-1} F\left(x^{*}, t_{i}\right)\right. \\
& -\left(x_{k}-\alpha^{-1} G^{-1} F\left(x_{k}, t_{i}\right) \|_{G}\right. \\
\leq & \lambda_{\max }^{\frac{1}{2}} \lambda_{\min }^{-\frac{1}{2}} \frac{1}{N_{k}} \sum_{t_{i} \in T_{k}} \Phi\left(t_{i}\right)\left(\left\|x_{k}-x^{*}\right\|\right. \\
& \left.+\alpha^{-1}\left\|G^{-1}\right\|\left\|F\left(x_{k}, t_{i}\right)-F\left(x^{*}, t_{i}\right)\right\|\right) \\
& \stackrel{k \rightarrow \infty}{\longrightarrow} 0 .
\end{aligned}
$$


So

$$
\frac{\lambda}{N_{k}} \sum_{t_{i} \in T_{k}} \Phi\left(t_{i}\right)\left\|H\left(x^{*}, t_{i}\right)-H\left(x_{k}, t_{i}\right)\right\| \stackrel{k \rightarrow \infty}{\longrightarrow} 0
$$

On the other hand, by (21), (25), (32), and (33), we have

$$
\begin{aligned}
& \frac{1}{N_{k}} \sum_{t_{i} \in T_{k}} \Phi\left(t_{i}\right)\left\|F_{x t}^{\prime \prime}\left(x_{k}, t_{i}\right) H\left(x_{k}, t_{i}\right)-F_{x t}^{\prime \prime}\left(x^{*}, t_{i}\right) H\left(x^{*}, t_{i}\right)\right\| \\
= & \frac{1}{N_{k}} \sum_{t_{i} \in T_{k}} \Phi\left(t_{i}\right) \| F_{x t}^{\prime \prime}\left(x_{k}, t_{i}\right)\left(H\left(x_{k}, t_{i}\right)-H\left(x^{*}, t_{i}\right)\right) \\
& +\left(F_{x t}^{\prime \prime}\left(x_{k}, t_{i}\right)-F_{x t}^{\prime \prime}\left(x^{*}, t_{i}\right) H\left(x^{*}, t_{i}\right) \|\right. \\
\leq & \frac{1}{N_{k}} \sum_{t_{i} \in T_{k}} \Phi\left(t_{i}\right)\left(\| F _ { x t } ^ { \prime \prime } ( x _ { k } , t _ { i } ) \| \left(H\left(x_{k}, t_{i}\right)-H\left(x^{*}, t_{i}\right) \|\right.\right. \\
& \left.+\left\|F_{x t}^{\prime \prime}\left(x_{k}, t_{i}\right)-F_{x t}^{\prime \prime}\left(x^{*}, t_{i}\right)\right\|\left\|H\left(x^{*}, t_{i}\right)\right\|\right) \\
\leq & C \cdot \frac{1}{N_{k}} \sum_{t_{i} \in T_{k}} \Phi\left(t_{i}\right)\left(\|\left(H\left(x_{k}, t_{i}\right)-H\left(x^{*}, t_{i}\right) \|\right.\right. \\
& \left.+\left\|F_{x t}^{\prime \prime}\left(x_{k}, t_{i}\right)-F_{x t}^{\prime \prime}\left(x^{*}, t_{i}\right)\right\|\right) \\
& \stackrel{k \rightarrow \infty}{\longrightarrow} 0 .
\end{aligned}
$$

So

$$
\begin{aligned}
& \frac{\lambda}{N_{k}} \sum_{t_{i} \in T_{k}} \Phi\left(t_{i}\right) \| F_{x t}^{\prime \prime}\left(x_{k}, t_{i}\right) H\left(x_{k}, t_{i}\right) \\
& -F_{x t}^{\prime \prime}\left(x^{*}, t_{i}\right) H\left(x^{*}, t_{i}\right) \| \stackrel{k \rightarrow \infty}{\longrightarrow} 0
\end{aligned}
$$

Noting that $C \geq \sup \left\{\left\|x_{k}\right\|, k=1,2, \ldots\right\}$, from (24) and (33), it implies that

$$
\begin{aligned}
& \frac{1}{N_{k}} \sum_{t_{i} \in T_{k}} \Phi\left(t_{i}\right)\left\|F_{x t}^{\prime \prime}\left(x_{k}, t_{i}\right) x_{k}-F_{x t}^{\prime \prime}\left(x^{*}, t_{i}\right) x^{*}\right\| \\
\leq & \frac{1}{N_{k}} \sum_{t_{i} \in T_{k}} \Phi\left(t_{i}\right)\left(\left\|F_{x t}^{\prime \prime}\left(x_{k}, t_{i}\right) x_{k}-F_{x t}^{\prime \prime}\left(x^{*}, t_{i}\right)\right\|\left\|x_{k}\right\|\right) \\
& +\left\|F_{x t}^{\prime \prime}\left(x^{*}, \xi_{i}\right)\right\|\left\|x_{k}-x^{*}\right\| \\
\leq & C \cdot \frac{1}{N_{k}} \sum_{t_{i} \in T_{k}} \Phi\left(t_{i}\right)\left(\left\|F_{x t}^{\prime \prime}\left(x_{k}, t_{i}\right)-F_{x t}^{\prime \prime}\left(x^{*}, t_{i}\right)\right\|+\left\|x_{k}-x^{*}\right\|\right) \\
& \stackrel{k \rightarrow \infty}{\longrightarrow} 0 .
\end{aligned}
$$

So

$$
\frac{\lambda}{N_{k}} \sum_{t_{i} \in T_{k}} \Phi\left(t_{i}\right)\left\|F_{x t}^{\prime \prime}\left(x_{k}, t_{i}\right) x_{k}-F_{x t}^{\prime \prime}\left(x^{*}, t_{i}\right) x^{*}\right\| \stackrel{k \rightarrow \infty}{\longrightarrow} 0 .
$$


By the same way as (33), we have

$$
\lim _{k \rightarrow 0} \frac{\lambda}{N_{k}} \sum_{t_{i} \in T_{k}} \Phi\left(t_{i}\right)\left\|H_{t}^{\prime}\left(x_{k}, t_{i}\right)-H_{t}^{\prime}\left(x^{*}, t_{i}\right)\right\|=0 .
$$

Thus, from (20), (26), (31), and (34), we can get

$$
\begin{aligned}
& \frac{1}{N_{k}} \sum_{t_{i} \in T_{k}} \Phi\left(t_{i}\right)\left\|F_{x}^{\prime}\left(x_{k}, t_{i}\right) H_{t}^{\prime}\left(x_{k}, t_{i}\right)-F_{x}^{\prime}\left(x^{*}, t_{i}\right) H_{t}^{\prime}\left(x^{*}, t_{i}\right)\right\| \\
= & \frac{1}{N_{k}} \sum_{t_{i} \in T_{k}} \Phi\left(t_{i}\right) \| F_{x}^{\prime}\left(x_{k}, t_{i}\right)\left(H_{t}^{\prime}\left(x_{k}, t_{i}\right)-H_{t}^{\prime}\left(x^{*}, t_{i}\right)\right) \\
& +\left(F_{x}^{\prime}\left(x_{k}, t_{i}\right)-F_{x}^{\prime}\left(x^{*}, t_{i}\right)\left\|H_{t}^{\prime}\left(x^{*}, t_{i}\right)\right\|\right. \\
\leq & \frac{1}{N_{k}} \sum_{t_{i} \in T_{k}} \Phi\left(t_{i}\right)\left(\| F _ { x } ^ { \prime } ( x _ { k } , t _ { i } ) \| \left(H_{t}^{\prime}\left(x_{k}, t_{i}\right)-H_{t}^{\prime}\left(x^{*}, t_{i}\right) \|\right.\right. \\
& \left.+\left\|F_{x}^{\prime}\left(x_{k}, t_{i}\right)-F_{x}^{\prime}\left(x^{*}, t_{i}\right)\right\|\left\|H_{t}^{\prime}\left(x^{*}, t_{i}\right)\right\|\right) \\
\leq & C \cdot \frac{1}{N_{k}} \sum_{t_{i} \in T_{k}} \Phi\left(t_{i}\right)\left(\|\left(H_{t}^{\prime}\left(x_{k}, t_{i}\right)\right.\right. \\
& \left.-H_{t}^{\prime}\left(x^{*}, t_{i}\right)\|+\| F_{x}^{\prime}\left(x_{k}, t_{i}\right)-F_{x}^{\prime}\left(x^{*}, t_{i}\right) \|\right) \\
& \stackrel{k \rightarrow \infty}{\longrightarrow} 0 .
\end{aligned}
$$

Hence

$$
\begin{aligned}
& \frac{\lambda}{N_{k}} \sum_{t_{i} \in T_{k}} \Phi\left(t_{i}\right)\left\|F_{x}^{\prime}\left(x_{k}, t_{i}\right) H_{t}^{\prime}\left(x_{k}, t_{i}\right)-F_{x}^{\prime}\left(x^{*}, t_{i}\right) H_{t}^{\prime}\left(x^{*}, t_{i}\right)\right\| \\
& \stackrel{k \rightarrow \infty}{\longrightarrow} 0 .
\end{aligned}
$$


Through the above analysis, our main purpose is to prove the conclusion of $\nabla \theta_{k}\left(x_{k}\right)=\nabla \theta_{k}\left(x^{*}\right)$, in order to prove $\nabla \theta_{k}\left(x_{k}\right)=\nabla \theta\left(x^{*}\right)$. By (5) and (33)-(35), we have following naturally

$$
\begin{aligned}
& \left\|\nabla \theta_{k}\left(x_{k}\right)-\nabla \theta\left(x^{*}\right)\right\| \\
& =\|\left.\lambda g_{x}^{\prime}\left(x_{k}, t\right) \Phi(t)\right|_{t \in T}-\frac{\lambda}{N_{k}} \sum_{t_{i} \in T_{k}} \Phi\left(t_{i}\right) g_{x t}^{\prime \prime}\left(x_{k}, t_{i}\right) \\
& +\left.(1-\lambda) 2 g g_{x}^{\prime}\left(x_{k}, t\right) \Phi(t)\right|_{t \in T} \\
& -\frac{1-\lambda}{N_{k}} \sum_{t_{i} \in T_{k}} \Phi\left(t_{i}\right)\left(g_{t}^{\prime} g_{x}^{\prime}+g g_{x t}^{\prime \prime}\right)\left(x_{k}, t_{i}\right) \\
& -\left.\lambda g_{x}^{\prime}\left(x^{*}, t\right) \Phi(t)\right|_{t \in T}+\frac{\lambda}{N_{k}} \sum_{t_{i} \in T_{k}} \Phi\left(t_{i}\right) g_{x t}^{\prime \prime}\left(x^{*}, t_{i}\right) \| \\
& -\left.(1-\lambda) 2 g g_{x}^{\prime}\left(x_{k}, t\right) \Phi(t)\right|_{t \in T} \\
& +\frac{1-\lambda}{N_{k}} \sum_{t_{i} \in T_{k}} \Phi\left(t_{i}\right)\left(g_{t}^{\prime} g_{x}^{\prime}+g g_{x t}^{\prime \prime}\right)\left(x^{*}, t_{i}\right) \| \\
& \leq\left|\lambda g_{x}^{\prime}\left(x_{k}, t\right) \Phi(t)\right|_{t \in T}-\left.\lambda g_{x}^{\prime}\left(x^{*}, t\right) \Phi(t)\right|_{t \in T} \mid \\
& +\left|(1-\lambda) 2 g g_{x}^{\prime}\left(x_{k}, t\right) \Phi(t)\right|_{t \in T} \\
& -\left.(1-\lambda) 2 g g_{x}^{\prime}\left(x^{*}, t\right) \Phi(t)\right|_{t \in T} \mid \\
& +\| \frac{\lambda}{N_{k}} \sum_{t_{i} \in T_{k}} \Phi\left(t_{i}\right) g_{x t}^{\prime \prime}\left(x_{k}, t_{i}\right) \\
& -\frac{\lambda}{N_{k}} \sum_{t_{i} \in T_{k}} \Phi\left(t_{i}\right) g_{x t}^{\prime \prime}\left(x^{*}, t_{i}\right) \| \\
& +\| \frac{1-\lambda}{N_{k}} \sum_{t_{i} \in T_{k}} \Phi\left(t_{i}\right)\left(g_{t}^{\prime} g_{x}^{\prime}+g g_{x t}^{\prime \prime}\right)\left(x_{k}, t_{i}\right) \\
& -\frac{1-\lambda}{N_{k}} \sum_{t_{i} \in T_{k}} \Phi\left(t_{i}\right)\left(g_{t}^{\prime} g_{x}^{\prime}+g g_{x t}^{\prime \prime}\right)\left(x^{*}, t_{i}\right) \| \\
& \leq\left|\lambda g_{x}^{\prime}\left(x_{k}, t\right) \Phi(t)\right|_{t \in T}-\left.\lambda g_{x}^{\prime}\left(x^{*}, t\right) \Phi(t)\right|_{t \in T} \mid \\
& +\left|(1-\lambda) 2 g g_{x}^{\prime}\left(x_{k}, t\right) \Phi(t)\right|_{t \in T} \\
& -\left.(1-\lambda) 2 g g_{x}^{\prime}\left(x^{*}, t\right) \Phi(t)\right|_{t \in T} \mid \\
& +\left\|\frac{\lambda}{N_{k}} \sum_{t_{i} \in T_{k}} \Phi\left(t_{i}\right)\left(g_{x t}^{\prime \prime}\left(x_{k}, t_{i}\right)-g_{x t}^{\prime \prime}\left(x^{*}, t_{i}\right)\right)\right\| \\
& +\| \frac{1-\lambda}{N_{k}} \sum_{t_{i} \in T_{k}} \Phi\left(t_{i}\right)\left(\left(g_{t}^{\prime} g_{x}^{\prime}+g g_{x t}^{\prime \prime}\right)\left(x_{k}, t_{i}\right)\right. \\
& \left.-\left(g_{t}^{\prime} g_{x}^{\prime}+g g_{x t}^{\prime \prime}\right)\left(x^{*}, t_{i}\right)\right) \| \\
& \leq\left|\lambda g_{x}^{\prime}\left(x_{k}, t\right) \Phi(t)\right|_{t \in T}-\left.\lambda g_{x}^{\prime}\left(x^{*}, t\right) \Phi(t)\right|_{t \in T} \mid \\
& +\left|(1-\lambda) g_{x}^{\prime}\left(x_{k}, t\right) \Phi(t)\right|_{t \in T}-\left.(1-\lambda) g_{x}^{\prime}\left(x^{*}, t\right) \Phi(t)\right|_{t \in T} \mid \\
& +\| \frac{\lambda}{N_{k}} \sum_{t_{i} \in T_{k}} \Phi\left(t_{i}\right)\left(\left[F_{t}^{\prime}\left(x_{k}, t_{i}\right)-\lambda F_{x t}^{\prime \prime}\left(x_{k}, t_{i}\right)\left(H\left(x_{k}, t_{i}\right)-x_{k}\right)\right.\right. \\
& \left.-\lambda\left(F_{x}^{\prime}\left(x_{k}, t_{i}\right)-\alpha G\right) H_{t}^{\prime}\left(x_{k}, t_{i}\right)\right] \\
& -\lambda\left[F_{t}^{\prime}\left(x^{*}, t_{i}\right)-F_{x t}^{\prime \prime}\left(x^{*}, t_{i}\right)\left(H\left(x^{*}, t_{i}\right)-x^{*}\right)\right. \\
& \left.\left.-\lambda\left(F_{x}^{\prime}\left(x^{*}, t_{i}\right)-\alpha G\right) H_{t}^{\prime}\left(x^{*}, t_{i}\right)\right]\right) \| \\
& +\| \frac{1-\lambda}{N_{k}} \sum_{t_{i} \in T_{k}} \Phi\left(t_{i}\right)\left(\left(g_{t}^{\prime} g_{x}^{\prime}+g g_{x t}^{\prime \prime}\right)\left(x_{k}, t_{i}\right)\right. \\
& \left.-\left(g_{t}^{\prime} g_{x}^{\prime}+g g_{x t}^{\prime \prime}\right)\left(x^{*}, t_{i}\right)\right) \| \text {. }
\end{aligned}
$$


While

$$
\begin{aligned}
& \| \frac{1-\lambda}{N_{k}} \sum_{t_{i} \in T_{k}} \Phi\left(t_{i}\right)\left(\left(g_{t}^{\prime} g_{x}^{\prime}+g g_{x t}^{\prime \prime}\right)\left(x_{k}, t_{i}\right)\right. \\
& \left.-\left(g_{t}^{\prime} g_{x}^{\prime}+g g_{x t}^{\prime \prime}\right)\left(x^{*}, t_{i}\right)\right) \| \\
& =\| \frac{1-\lambda}{N_{k}} \sum_{t_{i} \in T_{k}} \Phi\left(t_{i}\right)\left(\left(g_{t}^{\prime} g_{x}^{\prime}\left(x_{k}, t_{i}\right)\right.\right. \\
& \left.\left.-g_{t}^{\prime} g_{x}^{\prime}\left(x^{*}, t_{i}\right)\right)+\left(g g_{x t}^{\prime \prime}\left(x_{k}, t_{i}\right)-g g_{x t}^{\prime \prime}\left(x^{*}, t_{i}\right)\right)\right) \| .
\end{aligned}
$$

and

$$
\begin{aligned}
& \left\|\left(g_{t}^{\prime} g_{x}^{\prime}\left(x_{k}, t_{i}\right)-g_{t}^{\prime} g_{x}^{\prime}\left(x^{*}, t_{i}\right)\right)\right\| \\
= & \|\left(g_{t}^{\prime}\left(x_{k}, t_{i}\right) g_{x}^{\prime}\left(x_{k}, t_{i}\right)-g_{t}^{\prime}\left(x_{k}, t_{i}\right) g_{x}^{\prime}\left(x^{*}, t_{i}\right)\right) \\
& +\left(g_{t}^{\prime}\left(x_{k}, t_{i}\right) g_{x}^{\prime}\left(x^{*}, t_{i}\right)-g_{t}^{\prime}\left(x^{*}, t_{i}\right) g_{x}^{\prime}\left(x^{*}, t_{i}\right)\right) \| \\
\leq & \left\|g_{t}^{\prime}\left(x_{k}, t_{i}\right)\right\|\left\|g_{x}^{\prime}\left(x_{k}, t_{i}\right)-g_{x}^{\prime}\left(x^{*}, t_{i}\right)\right\| \\
& \left.+\left\|g_{t}^{\prime}\left(x_{k}, t_{i}\right)-g_{t}^{\prime}\left(x^{*}, t_{i}\right)\right\| \| g_{x}^{\prime}\left(x^{*}, t_{i}\right)\right) \| .
\end{aligned}
$$

Moreover, we have from the mean-value theorem that, for each $x_{k}$ and each $\xi_{i}$, there exists $\eta^{k i}=\alpha_{k i} x_{k}+\left(1-\alpha_{k i}\right) x^{*} \in B$ with $\alpha_{k i} \in[0,1]$ such that

$$
\left\|g_{t}^{\prime}\left(x_{k}, t_{i}\right)-g_{t}^{\prime}\left(x^{*}, t_{i}\right)\right\|=g_{t x}^{\prime \prime}\left(\eta_{k i}, t_{i}\right)\left(x_{k}-x^{*}\right),
$$

and

$$
\begin{aligned}
& \left\|g_{x}^{\prime}\left(x_{k}, t_{i}\right)-g_{x}^{\prime}\left(x^{*}, t_{i}\right)\right\| \\
& \leq\left\|F\left(x_{k}, t_{i}\right)-F\left(x^{*}, t_{i}\right)\right\| \\
& +\left\|F_{x}^{\prime}\left(x_{k}, t_{i}\right) H_{t}^{\prime}\left(x_{k}, t_{i}\right)-F_{x}^{\prime}\left(x^{*}, t_{i}\right) H_{t}^{\prime}\left(x^{*}, t_{i}\right)\right\| \\
& +\left\|F_{x}^{\prime}\left(x_{k}, t_{i}\right) x_{k}-F_{x}^{\prime}\left(x^{*}, t_{i}\right) x^{*}\right\| \\
& \alpha\|G\|\left\|H\left(x_{k}, t_{i}\right)-H\left(x^{*}, t_{i}\right)\right\|+\alpha\|G\|\left\|x_{k}-x^{*}\right\|,
\end{aligned}
$$

and

$$
\begin{aligned}
& \left\|F_{x}^{\prime}\left(x_{k}, t_{i}\right) H_{t}^{\prime}\left(x_{k}, t_{i}\right)-F_{x}^{\prime}\left(x^{*}, t_{i}\right) H_{t}^{\prime}\left(x^{*}, t_{i}\right)\right\| \\
= & \| F_{x}^{\prime}\left(x_{k}, t_{i}\right)\left(H_{t}^{\prime}\left(x_{k}, t_{i}\right)-H_{t}^{\prime}\left(x^{*}, t_{i}\right)\right) \\
& +\left(F_{x}^{\prime}\left(x_{k}, t_{i}\right)-F_{x}^{\prime}\left(x^{*}, t_{i}\right)\right) H_{t}^{\prime}\left(x^{*}, t_{i}\right) \| \\
\leq & \left\|F_{x}^{\prime}\left(x_{k}, t_{i}\right)\right\|\left\|H_{t}^{\prime}\left(x_{k}, t_{i}\right)-H_{t}^{\prime}\left(x^{*}, t_{i}\right)\right\| \\
& +\left\|F_{x}^{\prime}\left(x_{k}, t_{i}\right)-F_{x}^{\prime}\left(x^{*}, t_{i}\right)\right\|\left\|H_{t}^{\prime}\left(x^{*}, t_{i}\right)\right\|,
\end{aligned}
$$

and

$$
\begin{aligned}
& \left\|F_{x}^{\prime}\left(x_{k}, t_{i}\right) x_{k}-F_{x}^{\prime}\left(x^{*}, t_{i}\right) x^{*}\right\| \\
= & \|\left(F_{x}^{\prime}\left(x_{k}, t_{i}\right)-F_{x}^{\prime}\left(x^{*}, t_{i}\right)\right) x_{k} \\
& +F_{x}^{\prime}\left(x_{k}, t_{i}\right)\left(x_{k}-x^{*}\right) \| \\
\leq & \left\|F_{x}^{\prime}\left(x_{k}, t_{i}\right)-F_{x}^{\prime}\left(x^{*}, t_{i}\right)\right\| x_{k} \| \\
& +\left\|F_{x}^{\prime}\left(x_{k}, t_{i}\right)\right\|\left\|\left(x_{k}-x^{*}\right)\right\|,
\end{aligned}
$$


and also

$$
\begin{aligned}
& \left\|\left(g g_{x t}^{\prime \prime}\left(x_{k}, t_{i}\right)-g g_{x t}^{\prime \prime}\left(x^{*}, t_{i}\right)\right)\right\| \\
= & \|\left(g\left(x_{k}, t_{i}\right) g_{x t}^{\prime \prime}\left(x_{k}, t_{i}\right)-g\left(x_{k}, t_{i}\right) g_{x t}^{\prime \prime}\left(x^{*}, t_{i}\right)\right) \\
& +\left(g\left(x_{k}, t_{i}\right) g_{x t}^{\prime \prime}\left(x^{*}, t_{i}\right)-g\left(x^{*}, t_{i}\right) g_{x t}^{\prime \prime}\left(x^{*}, t_{i}\right)\right) \| \\
\leq & \left\|g\left(x_{k}, t_{i}\right)\right\|\left\|g_{x t}^{\prime \prime}\left(x_{k}, t_{i}\right)-g_{x t}^{\prime \prime}\left(x^{*}, t_{i}\right)\right\| \\
& \left.+\left\|g\left(x_{k}, t_{i}\right)-g\left(x^{*}, t_{i}\right)\right\| \| g_{x t}^{\prime \prime}\left(x^{*}, t_{i}\right)\right) \|,
\end{aligned}
$$

also there exists $z_{k i}=\beta_{k i} x_{k}+\left(1-\beta_{k i}\right) x^{*} \in B$ with $\beta_{k i} \in[0,1]$ such that

$$
\left\|g\left(x_{k}, t_{i}\right)-g\left(x^{*}, t_{i}\right)\right\|=g_{x}^{\prime}\left(z_{k i}, t_{i}\right)\left(x_{k}-x^{*}\right) .
$$

Through the above discussion and analysis, we get

$$
\left\|\nabla \theta_{k}\left(x_{k}\right)-\nabla \theta_{k}\left(x^{*}\right)\right\| \stackrel{k \rightarrow \infty}{\longrightarrow} 0,
$$

through integration by parts, we also know that

$$
\begin{aligned}
\nabla \theta\left(x^{*}\right) & =E\left[\nabla_{x}\left(\lambda g\left(x^{*}, \xi\right)+(1-\lambda) g^{2}\left(x^{*}, \xi\right)\right)\right] \\
& =\lambda E\left[\nabla_{x} g\left(x^{*}, \xi\right)\right]+(1-\lambda) E\left[\nabla_{x} g^{2}\left(x^{*}, \xi\right)\right] \\
& =\lambda E\left[\nabla_{x} g\left(x^{*}, \xi\right)\right]+2(1-\lambda) E\left[g\left(x^{*}, \xi\right) g^{\prime}\left(x^{*}, \xi\right)\right],
\end{aligned}
$$

and

$$
\begin{aligned}
& E\left[\nabla_{x} g\left(x^{*}, \xi\right)\right]=\int_{T} \nabla_{x} g\left(x^{*}, t\right) d \Phi(t) \\
= & \left.\nabla_{x} g\left(x^{*}, t\right) \Phi(t)\right|_{t \in T}-\int_{T} \Phi(t) d \nabla_{x} g\left(x^{*}, t\right) \\
= & \left.\nabla_{x} g\left(x^{*}, t\right) \Phi(t)\right|_{t \in T}-\int_{T} \Phi(t) g_{x t}^{\prime \prime}\left(x^{*}, t\right) d t,
\end{aligned}
$$

and

$$
\begin{aligned}
& E\left[g\left(x^{*}, \xi\right) g^{\prime}\left(x^{*}, \xi\right)\right]=\int_{T} g\left(x^{*}, t\right) g^{\prime}\left(x^{*}, t\right) d \Phi(t) \\
& =\left.g\left(x^{*}, t\right) g^{\prime}\left(x^{*}, t\right) \Phi(t)\right|_{t \in T}-\int_{T} \Phi(t) d g\left(x^{*}, t\right) g^{\prime}\left(x^{*}, t\right) \\
& =\left.g\left(x^{*}, t\right) g^{\prime}\left(x^{*}, t\right) \Phi(t)\right|_{t \in T}-\int_{T} \Phi(t) d g\left(x^{*}, t\right) g^{\prime}\left(x^{*}, t\right) \\
& =\left.g\left(x^{*}, t\right) g^{\prime}\left(x^{*}, t\right) \Phi(t)\right|_{t \in T} \\
& -\int_{T} \Phi(t)\left(\left(g^{\prime}\left(x^{*}, t\right)\right)^{2}+g\left(x^{*}, t\right) g^{\prime \prime}\left(x^{*}, t\right)\right) d t .
\end{aligned}
$$

Notice that

$$
\begin{aligned}
& \theta_{k}\left(x^{*}\right)=\left.\lambda \nabla_{x} g\left(x^{*}, t\right) \Phi(t)\right|_{t \in T}-\frac{\lambda}{N_{k}} \sum_{t_{i} \in T_{k}} \Phi\left(t_{i}\right) g^{\prime \prime}\left(x^{*}, t_{i}\right) \\
& +\left.2(1-\lambda) g\left(x^{*}, t\right) g^{\prime}\left(x^{*}, t\right) \Phi(t)\right|_{t \in T} \\
& -\frac{2(1-\lambda)}{N_{k}} \sum_{t_{i} \in T_{k}} \Phi\left(t_{i}\right)\left(\left(g^{\prime}\left(x^{*}, t_{i}\right)\right)^{2}+g\left(x^{*}, t_{i}\right) g^{\prime \prime}\left(x^{*}, t_{i}\right)\right),
\end{aligned}
$$


Owing to

$$
\lim _{k \rightarrow \infty} \frac{1}{N_{k}} \sum_{t_{i} \in T_{k}} \Phi\left(t_{i}\right) g_{x t}^{\prime \prime}\left(x^{*}, t_{i}\right)=\int_{T} \Phi(t) g_{x t}^{\prime \prime}\left(x^{*}, t\right) d t
$$

and

$$
\begin{aligned}
& \lim _{k \rightarrow \infty} \frac{1}{N_{k}} \sum_{t_{i} \in T_{k}} \Phi\left(t_{i}\right)\left(\left(g^{\prime}\left(x^{*}, t_{i}\right)\right)^{2}+g\left(x^{*}, t_{i}\right) g^{\prime \prime}\left(x^{*}, t_{i}\right)\right), \\
& =\int_{T} \Phi(t)\left(\left(g^{\prime}\left(x^{*}, t\right)\right)^{2}+g\left(x^{*}, t\right) g^{\prime \prime}\left(x^{*}, t\right)\right) d t .
\end{aligned}
$$

So it is easy to see that $\lim _{k \rightarrow \infty} \nabla \theta_{k}\left(x^{*}\right)=\nabla \theta\left(x^{*}\right)$. Thus, It is clear that $\lim _{k \rightarrow \infty} \nabla \theta_{k}\left(x_{k}\right)=\nabla \theta\left(x^{*}\right)$.

Definition 6. Suppose that $S=\left\{x \in R^{n} \mid c(x) \leq 0\right\}$, where $c_{i}: \mathbb{R}^{n} \rightarrow \mathbb{R}, i=1,2, \ldots, m$, are all continuously differentiable convex functions. A point $x_{k}$ is said to be stationary point of (12) if there exists a Lagrange multiplier vector $\mu_{k} \in \mathbb{R}^{m}$ such that

$$
\begin{gathered}
\nabla \theta_{k}\left(x_{k}\right)+\sum_{i=1}^{m}\left(\mu_{i}\right)_{k} \nabla c_{i}\left(x_{k}\right)=0, \\
0 \leq \mu_{k}, c\left(x_{k}\right) \leq 0 \text { and }\left(\mu_{k}\right)^{T} c\left(x_{k}\right)=0 .
\end{gathered}
$$

$x^{*}$ is said to be a stationary point of (10) if there exists a Lagrange multiplier vector $\mu^{*} \in R^{m}$ such that

$$
\begin{gathered}
\nabla \theta\left(x^{*}\right)+\sum_{i=1}^{m} \mu_{i}^{*} \nabla c_{i}\left(x^{*}\right)=0, \\
0 \leq \mu^{*}, c\left(x^{*}\right) \leq 0 \text { and }\left(\mu^{*}\right)^{T} c\left(x^{*}\right)=0 .
\end{gathered}
$$

Definition 7. The Slater's constraint qualification holds if there exists a vector $y \in R^{n}$ such that $c_{i}(y)<0$ for each $i=1,2, \ldots, m$.

Theorem 6. Let $x_{k}$ be stationary point to (12) for each $k$ and $x^{*}$ be an accumulation point of $\left\{x_{k}\right\}$. If the Slater constraint qualification holds, then $x^{*}$ is stationary point to problem (10).

Proof. Without loss of generality, we assume that $\lim _{k \rightarrow \infty} x_{k}=x^{*}$. Let $\mu_{k}$ be the corresponding multiplier vector satisfying (36) and (37).

(i) We first show that the sequence $\left\{\mu_{k}\right\}$ is bounded. To this end, we denote

$$
v_{k}:=\sum_{i=1}^{m}\left(\mu_{i}\right)_{k}
$$

Let $\left\{\mu_{k}\right\}$ be unbounded, which means $\lim _{k \rightarrow \infty} v_{k}=+\infty$. Taking a subsequence, we may assume that the limits $\mu_{i}^{*}:=\lim _{k \rightarrow \infty} \frac{\left(\mu_{i}\right)_{k}}{v_{k}}(i=1,2, \ldots, m)$ exist. For every $i \notin Y\left(x^{*}\right):=\left\{i \mid c_{i}\left(x^{*}\right)=0,1 \leq i \leq m\right\}$, it holds $c_{i}\left(x^{*}\right) \leq 0$ by (37), further more $\left(\mu_{i}^{*}\right)^{T} c_{i}\left(x^{*}\right)=0$, it holds $\mu_{i}^{*}=0$. Then, from (40),

$$
\sum_{i \in Y\left(x^{*}\right)} \mu_{i}^{*}=\sum_{i=1}^{m} \mu_{i}^{*}=1 .
$$


Note that $\nabla c_{i}$ is continuous for each $i$ and $\left\{\nabla \theta_{k}\left(x_{k}\right)\right\}$ is convergent by theorem 5. Because of $\lim _{k \rightarrow \infty} v_{k}=$ $+\infty, \lim _{k \rightarrow \infty} \frac{\nabla \theta_{k}\left(x_{k}\right)}{v_{k}} \rightarrow 0$. Dividing (36) by $v_{k}$ and taking a limit, we obtain

$$
\sum_{i \in Y\left(x^{*}\right)} \mu_{i}^{*} \nabla c_{i}\left(x^{*}\right)=\sum_{i=1}^{m} \mu_{i}^{*} \nabla c_{i}\left(x^{*}\right)=0
$$

Owing to the Slater's constraint qualification, there exists a vector $y \in R^{n}$ such that $c_{i}(y)<0$ for each $i=1,2, \ldots, m$. Noting that each $c_{i}$ is convex, we have

$$
\begin{array}{r}
\left(y-x^{*}\right)^{T} \nabla c_{i}\left(x^{*}\right) \leq c_{i}(y)-c_{i}\left(x^{*}\right)=c_{i}(y)<0, \\
\forall i \in Y\left(x^{*}\right) .
\end{array}
$$

From (42) and $\mu_{i}^{*} \geq 0$ for each $i$ by (37), we get $\mu_{i}^{*} \nabla c_{i}\left(x^{*}\right)=0$. Furthermore, $\nabla c_{i}\left(x^{*}\right) \neq 0$ from (43), it implies that $\mu_{i}^{*}=0$ for each $i \in \mathrm{Y}\left(x^{*}\right)$. This contradicts (41). Hence $\left\{\mu_{k}\right\}$ is bounded.

(ii) By (i), then $\mu_{k}$ must exists a subsequence such that $\mu^{*}:=\lim _{k \rightarrow \infty} \mu_{k}$, we still denote it as $\mu_{k}$. Note that both $c_{i}$ and $\nabla c_{i}$ are continuous for each $i$, by Theorem 5 , it holds

$$
\lim _{k \rightarrow \infty} \nabla \theta_{k}\left(x^{*}\right)=\nabla \theta\left(x^{*}\right)
$$

Taking a limit in (36) and (37), we obtain (38) and (39) immediately. Therefore, $x^{*}$ is stationary point to problem (10).

For the sake of completeness, we will propose a compact approximation approach for the case where $T$ is noncompact in the next section.

\section{Case Where Uncertain Event Space $T$ Is Unbounded}

The uncertain event space $T$ is supposed to be compact in the last section. From a technical point of view, it might be worthy to study whether approximation problem of (10) remain true under uncertain event space $T$ is unbounded. We now discuss the case where $T$ is an unbounded and closed subset of $R^{l}$ in this section. For this case, given a sufficiently large number $v$, we consider its compact approximation. we define a compact approximation of $T$ by

$$
T_{v}:=\{t \in T \mid\|t\| \leq v\}
$$

and consider the following approximation problem of (10):

$$
\begin{aligned}
& \min \theta_{v}(x):=E[g(x, \xi)]=\int_{T_{v}} g(x, t) d \Phi(t) \\
& \text { s.t. } x \in S .
\end{aligned}
$$

Since problem (44) has a compact uncertain set, we use the method proposed in the last section to solve (44). We make the following assumptions in this section:

(A1) The function $F(x, t)$ is Holder continuous in $x$ on $S$ with order $\tau>0$ and Holder constant $\kappa(t)$, which means

$$
\|F(x, t)-F(y, t)\| \leq \kappa(t)\|x-y\|^{\tau}, \forall x, y \in S .
$$

We further suppose that $E\left[\kappa^{2}(\xi)\right]<+\infty$

(A2) Satisfying $E\left[\|F(x, \xi)\|^{4}\right]<+\infty, \forall x \in S$,

Theorem 7. Under the Assumptions (A1) and (A2), we have

$$
E\left[g^{2}(x, \xi)\right]<+\infty, \forall x \in S,
$$


Proof. Since $g(x, t) \geq 0$ for $t \in T$, it follows from (3) that

$$
\begin{aligned}
\frac{\alpha}{2}\|x-H(x, t)\|_{G}^{2} & \leq(x-H(x, t))^{T} F(x, t) \\
& \leq\|(x-H(x, t))\|\|F(x, t)\|
\end{aligned}
$$

From (7), it holds that

$$
\|(x-H(x, t))\|\|F(x, t)\| \leq \lambda_{\min }^{-\frac{1}{2}}\|x-H(x, t)\|_{G}\|F(x, t)\| .
$$

We then have

$$
\|x-H(x, t)\|_{G} \leq \frac{2}{\alpha} \lambda_{\min }^{-\frac{1}{2}}\|F(x, t)\|
$$

and hence

$$
\begin{aligned}
\|x-H(x, t)\| & \leq \lambda_{\min }^{-\frac{1}{2}}\|x-H(x, t)\|_{G} \\
& \leq \frac{2}{\alpha \lambda_{\text {min }}}\|F(x, t)\| .
\end{aligned}
$$

As a result, it holds

$$
\begin{aligned}
g^{2}(x, t)= & \left((x-H(x, t))^{T} F(x, t)-\frac{\alpha}{2}\|x-H(x, t)\|_{G}^{2}\right)^{2} \\
& \leq\left(\|x-H(x, t)\|\|F(x, t)\|+\frac{\alpha}{2}\|x-H(x, t)\|_{G}^{2}\right)^{2} \\
& \leq\left(\frac{4}{\alpha \lambda_{\text {min }}}\|F(x, t)\|^{2}\right)^{2} .
\end{aligned}
$$

So

$$
g^{2}(x, t) \leq \frac{16}{\alpha^{2} \lambda_{\min }^{2}}\|F(x, t)\|^{4}
$$

This, together with (44), implies that $E[g(x, \xi)]<+\infty$. We obtain the desired result.

Theorem 8. Let $x_{v}$ be an optimal solution of problem (44) for each $v$ and let $\tilde{x}$ be an accumulation point of $x_{v}$. Then, under the Assumptions (A1) and (A2), $\tilde{x}$ is an optimal solution of problem (10).

Proof. For simplicity, we assume that $\lim _{v \rightarrow \infty} x_{v}=\tilde{x}$. It is obvious that $\tilde{x} \in S$.

We first show that

$$
\lim _{v \rightarrow \infty} \theta_{v}\left(x_{v}\right)=\theta(\tilde{x}) .
$$

It holds that

$$
\begin{aligned}
& \left|\theta_{v}\left(x_{v}\right)-\theta_{v}(\tilde{x})\right| \\
= & \mid \lambda \int_{T_{v}} g\left(x_{v}, t\right) d \Phi(t)+(1-\lambda) \int_{T_{v}} g^{2}\left(x_{v}, t\right) d \Phi(t) \\
& -\lambda \int_{T_{v}} g(\tilde{x}, t) d \Phi(t)-(1-\lambda) \int_{T_{v}} g^{2}(\tilde{x}, t) d \Phi(t) \\
\leq & \lambda \int_{T}\left|g\left(x_{v}, t\right)-g(\tilde{x}, t)\right| d \Phi(t) \\
& +(1-\lambda) \int_{T}\left|g^{2}\left(x_{v}, t\right)-g^{2}(\tilde{x}, t)\right| d \Phi(t)
\end{aligned}
$$


Firstly, we prove $\lambda \int_{T}\left|g\left(x_{v}, t\right)-g(\tilde{x}, t)\right| d \Phi(t) \rightarrow 0$.

$$
\begin{aligned}
& \left|\int_{T_{v}}\left(g\left(x_{v}, t\right)-g(\tilde{x}, t)\right) d \Phi(t)\right| \\
= & \mid \int_{T_{v}}\left(\left(x_{v}-H\left(x_{v}, t\right)\right)^{T} F\left(x_{v}, t\right)-\frac{\alpha}{2}\left\|x_{v}-H\left(x_{v}, t\right)\right\|_{G}^{2}\right. \\
& \left.-(\tilde{x}-H(\tilde{x}, t))^{T} F(\tilde{x}, t)+\frac{\alpha}{2}\|\tilde{x}-H(\tilde{x}, t)\|_{G}^{2}\right) d \Phi(t) \mid, \\
\leq & \int_{T}\left|\left(x_{v}-\tilde{x}+H(\tilde{x}, t)-H\left(x_{v}, t\right)\right)^{T} F\left(x_{v}, t\right)\right| d \Phi(t) \\
& +\int_{T}\left|(\tilde{x}-H(\tilde{x}, t))^{T}\left(F\left(x_{v}, t\right)-F(\tilde{x}, t)\right)\right| d \Phi(t) \\
& +\frac{\alpha}{2} \int_{T}|| \tilde{x}-H(\tilde{x}, t)\left\|_{G}^{2}-\right\| x_{v}-H\left(x_{v}, t\right) \|_{G}^{2} \mid d \Phi(t) .
\end{aligned}
$$

Next, we prove $\int_{T}\left|\left(x_{v}-\tilde{x}+H(\tilde{x}, t)-H\left(x_{v}, t\right)\right)^{T} F\left(x_{v}, t\right)\right| d \Phi(t) \rightarrow 0, \int_{T} \mid(\tilde{x}-H(\tilde{x}, t))^{T}\left(F\left(x_{v}, t\right)-\right.$ $F(\tilde{x}, t)) \mid d \Phi(t) \rightarrow 0$ and $\frac{\alpha}{2} \int_{T}\left|\|\tilde{x}-H(\tilde{x}, t)\|_{G}^{2}-\left\|x_{v}-H\left(x_{v}, t\right)\right\|_{G}^{2}\right| d \Phi(t) \rightarrow 0$, respectively. Before proving them, we have (48). By the nonexpansive property of $\operatorname{Proj}_{S, G}$, the holder continuity of $F$, and (7), we have

$$
\begin{aligned}
& \left\|H(\tilde{x}, t)-H\left(x_{v}, t\right)\right\|_{G} \\
& =\|\left(\tilde{x}-\alpha^{-1} G^{-1} F(\tilde{x}, t)-\left(x_{v}-\alpha^{-1} G^{-1} F\left(x_{v}, t\right) \|_{G}\right.\right. \\
& \leq \|\left(\tilde{x}-x_{v}\left\|_{G}+\right\| \alpha^{-1} G^{-1} F(\tilde{x}, t)-\alpha^{-1} G^{-1} F\left(x_{v}, t\right) \|_{G}\right. \\
& \leq \lambda_{\text {max }}^{\frac{1}{2}}\left(\left\|x_{v}-\tilde{x}\right\|+\alpha^{-1}\left\|G^{-1}\right\|\left\|F\left(x_{v}, t\right)-F(\tilde{x}, t)\right\|\right) \\
& \leq \sigma\left(\left\|x_{v}-\tilde{x}\right\|+\left\|x_{v}-\tilde{x}\right\|{ }^{\tau} \kappa(t)\right),
\end{aligned}
$$

where $\sigma:=\max \left\{\lambda_{\max }^{\frac{1}{2}}, \lambda_{\max }^{\frac{1}{2}} \alpha^{-1}\left\|G^{-1}\right\|\right\}$.

$$
\begin{aligned}
\left\|H(\tilde{x}, t)-H\left(x_{v}, t\right)\right\| & \leq \lambda_{\min }^{-\frac{1}{2}}\left\|H(\tilde{x}, t)-H\left(x_{v}, t\right)\right\|_{G} \\
& \leq \sigma \lambda_{\text {min }}^{-\frac{1}{2}}\left(\left\|x_{v}-\tilde{x}\right\|+\left\|x_{v}-\tilde{x}\right\|^{\tau} \mathcal{\kappa}(t)\right) .
\end{aligned}
$$

We have from Theorem 7 and the Cauchy-Schwarz inequality that, for any $x \in S$,

$$
E[\kappa(\xi)] \leq \sqrt{E\left[\kappa^{2}(\xi)\right]}<+\infty
$$

and

$$
E[\|F(x, \xi)\|] \leq \sqrt{E\left[\|F(x, \xi)\|^{2}\right]}<+\infty,
$$

and

$$
|E[\kappa(\xi)\|F(x, \xi)\|]| \leq \sqrt{E\left[\kappa^{2}(\xi)\right]} \sqrt{E\left[\|F(x, \xi)\|^{2}\right]}<+\infty .
$$


Therefore, we can infer that

$$
\begin{aligned}
& \int_{T}\left|\left(x_{v}-\tilde{x}+H(\tilde{x}, t)-H\left(x_{v}, t\right)\right)^{T} F\left(x_{v}, t\right)\right| d \Phi(t) \\
\leq & \int_{T} \mid\left(\left\|x_{v}-\tilde{x}\right\|+\left\|H(\tilde{x}, t)-H\left(x_{v}, t\right)\right\|\right) \\
& \times\left(\|F(\tilde{x}, t)\|+\left\|F(\tilde{x}, t)-F\left(x_{v}, t\right)\right\|\right) d \Phi(t) \\
\leq & \int_{T} \mid\left(\left(1+\sigma \lambda_{\min }^{-\frac{1}{2}}\right)\left\|x_{v}-\tilde{x}\right\|+\sigma \lambda_{\min }^{-\frac{1}{2}}\left\|x_{v}-\tilde{x}\right\|^{\tau} \mathcal{K}(t)\right) \\
& \times\left(\|F(\tilde{x}, t)\|+\left\|x_{v}-\tilde{x}\right\|^{\tau} \mathcal{K}(t) d \Phi(t)\right. \\
= & \left(1+\sigma \lambda_{\min }^{-\frac{1}{2}}\right)\left\|x_{v}-\tilde{x}\right\| E[\|F(\tilde{x}, \tilde{\xi})\|] \\
& +\sigma \lambda_{\min }^{-\frac{1}{2}}\left\|x_{v}-\tilde{x}\right\|^{\tau} E[\kappa(\tilde{\xi})\|F(\tilde{x}, \tilde{\xi})\|] \\
& +\left(1+\sigma \lambda_{\min }^{-\frac{1}{2}}\right)\left\|x_{v}-\tilde{x}\right\|^{\tau+1} E[\kappa(\tilde{\xi})] \\
& +\sigma \lambda_{\min }^{-\frac{1}{2}}\left\|x_{v}-\tilde{x}\right\|^{2 \tau} E\left[\kappa^{2}(\tilde{\xi})\right] \\
& \stackrel{v \rightarrow \infty}{\longrightarrow} 0,
\end{aligned}
$$

where the second inequality follows from (49) and the holder continuity of $F$.

Similarly, from Assumption (A1), Theorem 7, and (45)-(52), we obtain

$$
\begin{aligned}
& \int_{T}\left|(\tilde{x}-H(\tilde{x}, t))^{T}\left(F\left(x_{v}, t\right)-F(\tilde{x}, t)\right)\right| d \Phi(t) \\
& \leq \int_{T}\|\tilde{x}-H(\tilde{x}, t)\|\left\|F\left(x_{v}, t\right)-F(\tilde{x}, t)\right\| d \Phi(t) \\
& \leq \int_{T} \frac{2}{\alpha \lambda_{\min }}\|F(\tilde{x}, t)\|\left\|F\left(x_{v}, t\right)-F(\tilde{x}, t)\right\| d \Phi(t) \\
& \leq \frac{2}{\alpha \lambda_{\min }}\left\|x_{v}-\tilde{x}\right\|^{\tau} \int_{T} \kappa(t)\|F(\tilde{x}, t)\| d \Phi(t) \\
& =\frac{2}{\alpha \lambda_{\min }}\left\|x_{v}-\tilde{x}\right\|^{\tau} E[\kappa(t)\|F(\tilde{x}, t)\|] \\
& \stackrel{v \rightarrow \infty}{\longrightarrow} 0,
\end{aligned}
$$


and

$$
\begin{aligned}
& \left.\frac{\alpha}{2} \int_{T} \mid\|(\tilde{x}-H(\tilde{x}, t))\|_{G}^{2}-\| x_{v}-H\left(x_{v}, t\right)\right) \|_{G}^{2} \mid d \Phi(t) \\
= & \frac{\alpha}{2} \int_{T} \mid\left(\|\tilde{x}-H(\tilde{x}, t)\|_{G}+\left\|x_{v}-H\left(x_{v}, t\right)\right\|_{G}\right) \\
& \times\left(\|\tilde{x}-H(\tilde{x}, t)\|_{G}-\left\|x_{v}-H\left(x_{v}, t\right)\right\|_{G}\right) \mid d \Phi(t) \\
\leq & \frac{\alpha}{2} \int_{T}\left(\|\tilde{x}-H(\tilde{x}, t)\|_{G}+\left\|x_{v}-H\left(x_{v}, t\right)\right\|_{G}\right) \\
& \times\left\|\tilde{x}-H(\tilde{x}, t)-x_{v}+H\left(x_{v}, t\right)\right\|_{G} d \Phi(t) \\
\leq & \lambda_{\min }^{-\frac{1}{2}} \int_{T}\left(\|F(\tilde{x}, t)\|+\left\|F\left(x_{v}, t\right)\right\|\right) \\
& \times\left(\lambda_{\text {max }}^{\frac{1}{2}}\left\|x_{v}-\tilde{x}\right\|+\left\|H(\tilde{x}, t)-H\left(x_{v}, t\right)\right\|_{G}\right) d \Phi(t) \\
\leq & \lambda_{\min }^{-\frac{1}{2}} \int_{T}\left(2\|F(\tilde{x}, t)\|+\left\|x_{v}-\tilde{x}\right\|^{\tau} \mathcal{K}(t)\right) \\
& \times\left(\left(\lambda_{\text {max }}^{\frac{1}{2}}+\sigma\right)\left\|x_{v}-\tilde{x}\right\|+\sigma\left\|x_{v}-\tilde{x}\right\|^{\tau} \mathcal{K}(t)\right) d \Phi(t) \\
= & 2 \lambda_{\min }^{-\frac{1}{2}}\left(\lambda_{\text {max }}^{\frac{1}{2}}+\sigma\right)\left\|x_{v}-\tilde{x}\right\| E[\|F(\tilde{x}, \xi)\|] \\
& +2 \sigma \lambda_{\min }^{-\frac{1}{2}}\left\|x_{v}-\tilde{x}\right\|^{\tau} E[\kappa(\xi)\|F(\tilde{x}, \xi)\|] \\
& +\left(\lambda_{\min }^{-\frac{1}{2}}\left(\lambda_{\text {max }}^{\frac{1}{2}}+\sigma\right)\left\|x_{v}-\tilde{x}\right\|^{\tau+1} E[\kappa(\xi)]\right. \\
& \left.+\sigma \lambda_{\min }^{-\frac{1}{2}}\left\|x_{v}-\tilde{x}\right\|^{2 \tau} E\left[\kappa^{2}(\tilde{\xi})\right)\right] \\
& \stackrel{v \rightarrow \infty}{\longrightarrow} 0 .
\end{aligned}
$$

By consequence, it holds that

$$
\left|\int_{T}\left(g\left(x_{v}, t\right)-g(\tilde{x}, t)\right) d \Phi(t)\right| \stackrel{v \rightarrow \infty}{\longrightarrow} 0 .
$$

Then, we prove $(1-\lambda) \int_{T}\left|g^{2}\left(x_{v}, t\right)-g^{2}(\tilde{x}, t)\right| d \Phi(t) \rightarrow 0$.

By mean-value theorem, for each $x_{v}$ and each $\xi$, there exist $y_{v}=a_{v} x_{v}+\left(1-a_{v}\right) \tilde{x}$ with $a_{v} \in[0,1]$ such that

$$
g^{2}\left(x_{v}, t\right)-g^{2}(\tilde{x}, t)=2 g\left(y^{v}, \xi\right) g_{x}^{\prime}\left(y^{v}, \xi\right)^{T}\left(x^{v}-\tilde{x}\right),
$$

it follows that

$$
\left|g^{2}\left(x_{v}, t\right)-g^{2}(\tilde{x}, t)\right| \leq 2 g\left(y^{v}, \xi\right)\left\|g_{x}^{\prime}\left(y^{v}, \xi\right)^{T}\right\| \cdot\left\|\left(x^{v}-\tilde{x}\right)\right\| .
$$

Furthermore, in view of the continuity of $g, g_{x}^{\prime}$ and the compactness of $\left\{y^{v}\right\} \times T_{v}$, as $v \rightarrow \infty$, we have that

$$
(1-\lambda) \int_{T_{v}}\left|g^{2}\left(x_{v}, t\right)-g^{2}(\tilde{x}, t)\right| d \Phi(t) \rightarrow 0 .
$$

From (53) and (54), it holds that

$$
\left|\theta_{v}\left(x_{v}\right)-\theta_{v}(\tilde{x})\right| \stackrel{v \rightarrow \infty}{\longrightarrow} 0 .
$$

On the other hand, it is easy to see from Theorem 7 that

$$
\begin{aligned}
& \lim _{v \rightarrow \infty}\left(\theta_{v}(\tilde{x})-\theta(\tilde{x})\right) \\
& =\lim _{v \rightarrow \infty} \int_{T \backslash T_{v}}\left(\lambda g(\tilde{x}, t)+(1-\lambda) g^{2}(\tilde{x}, t)\right) d \Phi(t)=0 .
\end{aligned}
$$


Noting that

$$
\left|\theta_{v}\left(x_{v}\right)-\theta(\tilde{x})\right| \leq\left|\theta_{v}\left(x_{v}\right)-\theta_{v}(\tilde{x})\right|+\left|\theta_{v}(\tilde{x})-\theta(\tilde{x})\right|,
$$

we get (47) from (55) and (56) immediately.

(ii) Since $x_{v}$ is an optimal solution of problem (44) for each $v$, we have

$$
\theta_{v}\left(x_{v}\right) \leq \theta_{v}(x) \leq \theta(x), \forall x \in S
$$

Consequently, letting $v \rightarrow \infty$ in (57) and taking (47) into account, we obtain

$$
\theta(\tilde{x}) \leq \theta(x), \forall x \in S .
$$

This manifests that $\tilde{x}$ is an optimal solution of (10).

\section{Conclusions}

Based on the discussion of the previous section, we proposed a method of convex combined expectations of the least absolute deviation and least squares about the so-called regularized gap function for nonlinear uncertain variational inequality problems (for short, UNVIP). We succeeded in establishing the UWERM model and extend the results given in [14] to the case where the uncertain event space is compact. As shown in the paper, convergence of global optimal solutions and convergence of stationary points are analyzed respectively. Moreover, we present a compact approximation approach for the case where the uncertain event space is unbounded.

Author Contributions: All authors contributed equally and significantly in writing this article: writing-original draft preparation, Z.J., conceptualization, C.L. and M.P., investigation, Z.J., funding acquisition, C.L. All authors read and approved the final manuscript.

Funding: National Natural Science Foundation of China (No. 71561001), the Major Projects of North Minzu University (ZDZX201805), the Key Scientific Research Projects in 2017 at North Minzu University (2017KJ13) and First-Class Disciplines Foundation of Ningxia (Grant No. NXYLXK2017B09).

Acknowledgments: This work was supported by the National Natural Science Foundation of China (No. 71561001), the Major Projects of North Minzu University (ZDZX201805), the Key Scientific Research Projects in 2017 at North Minzu University (2017KJ13) and First-Class Disciplines Foundation of Ningxia (Grant No. NXYLXK2017B09).

Conflicts of Interest: The authors declare no conflict of interest.

\section{References}

1. Yao, J.C. The generalized quasi-variational inequality problem with applications. J. Math. Anal. Appl. 1991, 158, 139-160. [CrossRef]

2. Yao, J.; Guo, J. Variational and generalized variational inequality with discontinuous mappings. J. Math. Anal. Appl. 1994 82, 371-392. [CrossRef]

3. Yang, X.Q.; Yao, J.C. Gap functions and fxistence of solutions to set-valued vector variational inequalities. J. Optim. Theory Appl. 2002, 115, 407-417. [CrossRef]

4. Mititelu, S.; Postolache, M. Efficiency and duality for multitime vector fractional variational problems on manifolds. Balk. J. Geom. Appl. 2011, 16, 90-101.

5. Yao, Y.H.; Postolache, M. Construction algorithms for a class of monotone variational inequalities. Optim. Lett. 2016, 10, 1519-1528. [CrossRef]

6. Thakur, B.S.; Postolache, M. Existence and approximation of solutions for generalized extended nonlinear variational inequalities. J. Inequal. Appl. 2013, 2013, 590. [CrossRef]

7. Yao, Y.; Postolache, M.; Liou, Y.C. Variant extragradient-type method for monotone variational inequalities. Fixed Point Theory Appl. 2013, 2013, 185. [CrossRef]

8. Yao, Y.H.; Chen, R.D.; Xu, H.K. Schemes for finding minimum-norm solutions of variational inequalities. Nonlinear Anal. 2010, 72, 3447-3456. [CrossRef] 
9. Yao, Y.H.; Liou, Y.C.; Yao, J.C. Iterative algorithms for the split variational inequality and fixed point problems under nonlinear transformations. J. Nonlinear Sci. Appl. 2017, 10, 843-854. [CrossRef]

10. Yao, Y.H.; Zheng, X.X. The split variational inequality problem and its algorithm iteration. J. Nonlinear Sci. Appl. 2017, 10, 2649-2661. [CrossRef]

11. Facchinei, F.; Pang, J.S. Finite-Dimensional Variational Inequalities and Complementarity Problems; Springer: New York, NY, USA, 2003.

12. Liu, B. Uncertainty Theory, 4th ed.; Springer: Berlin, Germany, 2015.

13. Chen, Q.Q.; Zhu, Y.G. A class of uncertain variational inequality problems. J. Inequal. Appl. 2015, $2015,231$. [CrossRef]

14. Li, C.L.; Jia, Z.F. Expected residual minimization method for uncertain variational inequality problems. J. Nonlinear Sci. Appl. 2017, 10, 5958-5975. [CrossRef]

15. Fukushima, M. Equivalent differentiable optimization problems and descent methods for asymmetric variational inequality problems. Math. Program. 1992, 53, 99-110. [CrossRef]

16. Niederreiter, H. Random Number Generation and Quasi-Monte Carlo Methods; SIAM: Philadelphia, PA, USA, 1992.

(C) 2019 by the authors. Licensee MDPI, Basel, Switzerland. This article is an open access article distributed under the terms and conditions of the Creative Commons Attribution (CC BY) license (http:// creativecommons.org/licenses/by/4.0/). 